ENSAYOS

\title{
ACERCA DEL PENSAMIENTO CAUSAL DINAMICO: HACIA UNA PERSPECTIVA EDUCATIVA
}

On dynamic causal thinking: towards an educational perspective

\author{
Martin Schaffernicht ${ }^{1}$ Patricio Madariaga ${ }^{2}$ \\ ${ }^{1}$ Facultad de Ciencias Empresariales, Universidad de Talca, Talca, Chile. \\ martin@utalca.cl \\ ${ }^{2}$ Dirección de Tecnologías de Aprendizaje, Universidad de Talca, \\ Avda. Lircay s/n, Talca, Chile.pmadar@utalca.cl
}

\section{Resumen}

En dinámica de sistemas, los vínculos causales entre variables indican dirección y "polaridad", lo que es difícil de aprender para estudiantes. La revisión de la definición "popular" de polaridad y la justificación de una definición más "completa" nos permiten señalar que pensamos en las variables en forma estática. Una aproximación dinámica nos conduce a distinguir un patrón de conducta general de la conducta particular debido a un evento. Encontramos un gran número de configuraciones de conducta general para casos con dos variables con un vínculo causal. Sólo las cuatro configuraciones con conducta general estática son bien clasificadas por la definición "popular"; la definición "completa" permite clasificar todas las configuraciones. Construimos un modelo conceptual de las configuraciones y cinco preguntas de investigación. Terminamos mostrando el uso educativo de las configuraciones.

Palabras clave: pensamiento causal, polaridad, complejidad dinámica.

\begin{abstract}
In system dynamics, causal links between variables indicate the direction and the polarity of influence. An examination of the "popular" definition of polarity and the justification of the "complete" definition suggest that people think of variables in a static manner. A dynamic approach leads to a distinction between the variables' basic behavior and their particular behavior due to one event. We find a large set of basic behavior configurations for two connected variables. The "popular" definition holds only for those 4 configurations that correspond to static basic behavior, but the "complete" definition classifies all of them appropriately. We propose a conceptual model of the configurations and 5 research challenges. We finish showing a design for educational use of the configurations.
\end{abstract}

Key words: causal model, polarity, dynamic complexity. 


\section{INTRODUCCION}

La dinámica de sistemas trata de cómo las cosas cambian en el tiempo y de cómo podemos actuar sin causar efectos laterales contradictorios (Sterman 2000). Se basa en la premisa que nuestro mundo contiene muchos bucles de retroalimentación, por los cuales las consecuencias de nuestras acciones nos vuelven a afectar; también asume que el mundo muchas veces nos sorprende con su complejidad dinámica. Esta disciplina hace un uso intensivo del concepto de "causa"; se asume que los cambios que ocurren en el mundo son gobernados por relaciones causales y que el actor humano puede diseñar políticas de decisión exitosas gracias al trabajo riguroso con sus propias atribuciones causales. En la literatura acerca de esta disciplina, predomina una visión de un mundo determinístico con múltiples causas, donde el estudio de las relaciones causales permite explicar y gobernar. No hay consenso en cuanto a si las relaciones causales pueden ser directamente percibidas o no, ni si existen en la realidad o solamente en nuestros modelos de ella (Pedercini 2006).

Para la dinámica de sistemas, la influencia causal tiene una "polaridad". Esto la distingue de otras disciplinas sistémicas que también usan el concepto de retroalimentación (Richardson 1991). La praxis de enseñar la dinámica de sistemas a estudiantes universitarios de pregrado nos ha mostrado que la noción de causalidad con polaridad es muy difícil de asimilar para los alumnos. Sin embargo, la buena comprensión de este concepto es fundamental para "hacer" dinámica de sistemas y -en forma generalincide en la comprensión de muchos fenómenos de cambio.

La revisión de la literatura muestra que no parece ser un tema mayor entre los especialistas: en el texto docente de referencia de Sterman (2000) se da una definición del concepto de polaridad en el contexto de los "diagramas de bucle causal". Se revela además que a pesar de la discusión crítica sobre la definición del concepto y su uso (Richardson 1997; Warren 2004) se sigue utilizando una definición problemática, que se analizará a continuación. No hay investigación empírica acerca de cómo pensamos sobre y con la polaridad, siendo la discusión en la literatura de naturaleza normativa (Richardson 1997; Sterman 2000).

Surge, entonces, la necesidad de investigar el concepto de la polaridad en las relaciones causales y cómo pensamos sobre esta relación entre dos variables, lo que proponemos en la siguiente forma.

En una primera sección se introduce brevemente el concepto y su representación usual, los diagramas causales. Se presenta la definición "popular" y se revisa la crítica desde la literatura; la definición "completa" del término permite salvar el concepto de la aparente contradicción. La argumentación sigue mostrando que cuando pensamos solamente en dos variables, otras quedan implícitas. A continuación se deriva entonces el que las variables no son estáticas, sino que tienen su propia dinámica y que el juicio intuitivo no parece tomar en cuenta. Nos encontramos con que, entre dos variables relacionadas causalmente de manera lineal ${ }^{1}$, hay no menos de 36 configuraciones de causalidad diferentes que la definición de "polaridad" debe permitir clasificar. La definición popular permite clasificar bien las configuraciones simples, pero no las complejas; en cambio, la definición completa permite clasificar uniformemente a todas las configuraciones.

1 Nos limitamos además a casos de desplazamiento de la curva, excluyendo cambios de pendiente. 
En la segunda sección mostramos que este aspecto del pensamiento estático es nuevo y cómo se relaciona con otros aspectos que han sido discutidos en la literatura. Luego proponemos cinco preguntas de investigación diferentes, y se muestra de qué forma un experimento con las 36 configuraciones puede ser usado para responder las preguntas y confrontar las hipótesis con datos empíricos.

La tercera sección muestra de qué manera proponemos insertar este tema en una lógica educativa, de forma a contribuir al pensamiento crítico desde la perspectiva dinámica.

Concluimos constatando que el mundo -cuyos atributos van cambiando- es más complejo de lo que sugieren los simples diagramas causales. Aparece una brecha entre la abstracción diagramática, el pensamiento intuitivo y estático y la complejidad dinámica de las situaciones que intentamos gobernar. Subrayamos la necesidad de realizar investigaciones en este ámbito.

\section{LA NATURALEZA DEL EFECTO CAUSAL EN UN MUNDO DINAMICO}

Causalidad y polaridad. Un mundo dinámico es uno en el cual "las cosas" cambian en el tiempo: cambian de lugar, de forma, de estructura. Cuando describimos "las cosas", hacemos uso de variables: nos fijamos en un determinado aspecto -relevante para un propósito determinado- e intentamos describir el valor que un objeto de observación presenta para tal o cual variable. En algunos casos podemos especificar escalas de valores ordinales, en otros casos escalas cardinales, pero en general somos capaces de indicar el orden entre valores particulares. Por ejemplo, si nos interesa la variable "tasa de interés", podemos cuantificar muy bien e indicar que 2,5\% > 2,3\%. Pero también cuando observamos los funcionarios de una organización para entender su grado de motivación podemos claramente distinguir entre "bueno" y "malo" y decir que "bueno" > "malo".

Acostumbramos razonar sobre las variables y los vínculos causales con los llamados "diagramas causales"2. En términos genéricos, un diagrama causal tiene la siguiente composición:

\section{Figura 1}

La notación del vínculo causal y algunos ejemplos

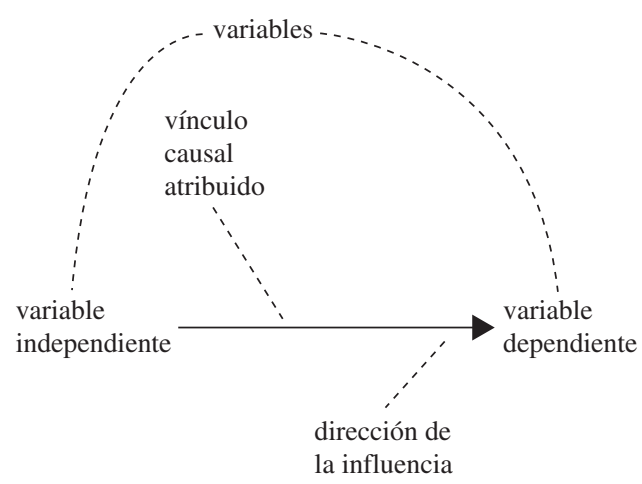

2 Para los efectos de nuestra argumentación aquí no necesitamos los "bucles". 
Necesitamos a lo menos dos variables y un vínculo causal entre ellas. Tenemos una variable independiente (que no depende de nada dentro del diagrama) y una variable dependiente, que depende evidentemente de la independiente. Esto es poco usual para la dinámica de sistemas, donde los bucles de retroalimentación juegan un rol importante; sin embargo, para nuestro propósito es conveniente limitarnos al caso de dos variables con un solo vínculo causal. El vínculo causal debe indicar la dirección de la influencia y su "polaridad".

Cuando pensamos en la conexión causal entre dos variables, lo hacemos frecuentemente del siguiente modo expresado a través de estos ejemplos ${ }^{3}$ : "si la dirección de la organización reduce el tiempo de vacaciones, entonces la motivación de los funcionarios baja" o "si el Banco Central baja la tasa de interés, entonces la construcción de viviendas aumenta". En el primero de estos casos, ambas variables bajan, por lo cual se dice que se mueven en el mismo sentido; en el segundo caso, se mueven en sentido opuesto. He aquí dos ejemplos:

Figura 2

Dos ejemplos de diagrama causal

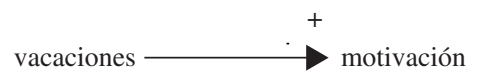

$\underset{\text { tasa de }}{\text { interés }} \longrightarrow$ viviendas

Para empezar a analizar lo que significa "polaridad" tenemos que volver a los cambios en las variables. Con aumentar, subir, bajar nos referimos a cambios o movimientos en las variables: hasta un cierto momento, la variable tuvo un valor, y luego tuvo otro más alto o más bajo. Así nos aparecen las 4 posibilidades que usualmente se muestran, ya que cada una de las dos variables puede subir o bajar $(2 \times 2=4$ posibilidades):

Figura 3

4 configuraciones de cambios para 2 tipos de polaridad

variable dependiente

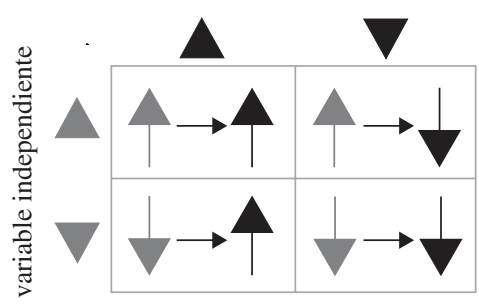

3 En los enunciados textuales, las variables aparecen en letras negritas y sus cambios o movimientos en letras cursivas. 
Definamos una codificación para referirnos a las configuraciones en el texto: hablaremos de "signo del cambio en la variable independiente/dependiente", el cual podrá ser:

- "positivo" (“u” para "up" en inglés) o

• "negativo" ("d" para "down" en inglés)

Tenemos entonces las posibilidades siguientes para los signos del cambio en cada una de las dos variables:

Tabla 1

Los signos de cambio en las dos variables

\begin{tabular}{|l|c|c|}
\hline Polaridad & \multicolumn{2}{|c|}{ Variable dependiente } \\
\hline Variable independiente & $\begin{array}{c}\text { Positivo-positivo } \\
\text { Negativo-positivo }\end{array}$ & $\begin{array}{c}\text { Positivo-negativo } \\
\text { Negativo-negativo }\end{array}$ \\
\hline
\end{tabular}

La definición "popular" de polaridad. En este contexto, aparece la definición "popular" de polaridad:

- Polaridad positiva: cuando la variable independiente cambia, entonces la variable dependiente cambia en el mismo sentido (símbolo "+" o "s" como "same" en inglés);

- Polaridad negativa: cuando la variable independiente cambia, entonces la variable dependiente cambia en el sentido opuesto (símbolo "--" u "o" como "opposit" en inglés).

Esta definición recibe aquí el apelativo "popular" porque se usa ampliamente en las publicaciones que usan "diagramas de bucle causal".

La definición nos recuerda que un diagrama causal es el resultado final de un proceso de abstracción en el cual queda solamente el nombre de las variables en forma explícita. Recordemos que debajo de estas abstracciones hay dos variables: atributos de algo, de los cuales se miden los valores y que van cambiando en el tiempo. La "causa" es un evento que interviene en los valores de la variable independiente y el "efecto" es el evento gatillado por el vínculo causal en la variable dependiente. Podemos entonces mirar con mayor detalle lo que pasa en las dos variables: 
Figura 4

Causa y efecto en la historia de las variables
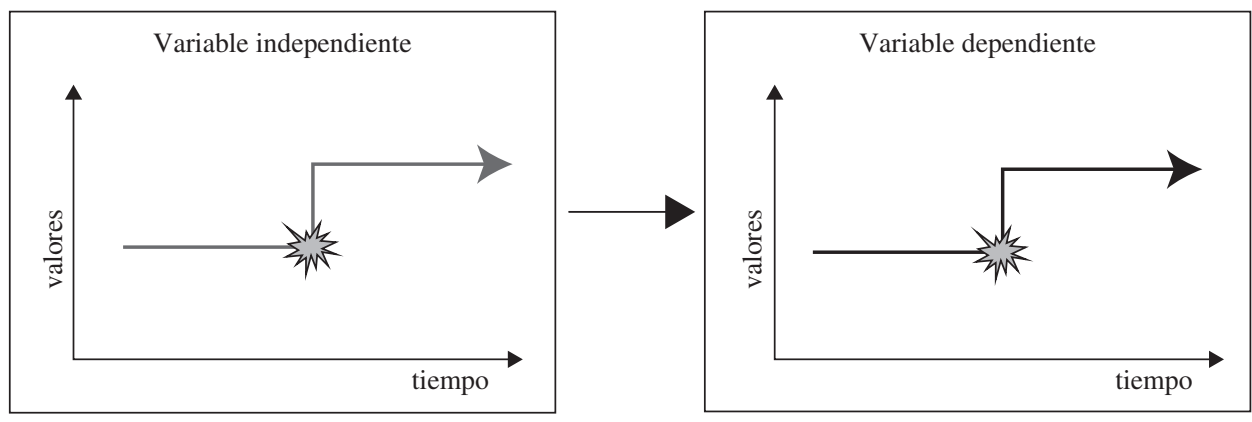

Vemos cómo el evento (la estrella en el diagrama) ha significado un cambio en cada una de las variables. El cambio ocurre en un determinado momento, y en los demás instantes no ocurren cambios.

Esto es lo que implícitamente imaginamos cuando se dice que "si la dirección de la organización aumenta el tiempo de vacaciones, entonces la motivación de los funcionarios sube", que podemos graficar de la siguiente manera:

Figura 5

Causa y efecto en un caso específico

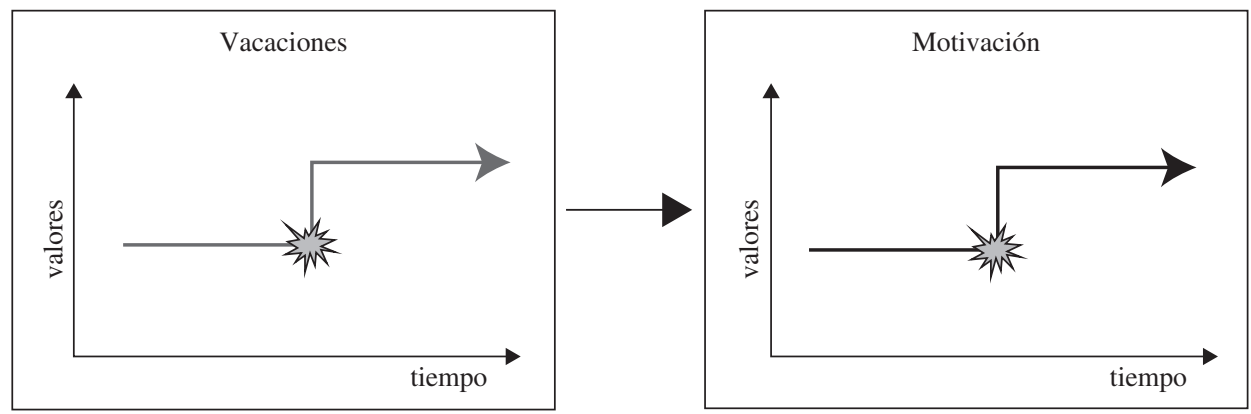

De esta manera, las 4 posibilidades tienen un supuesto implícito: cambio o movimiento ocurre solamente en los eventos de "causa" y de "efecto": 
Figura 6

Las 4 configuraciones tradicionales en el tiempo

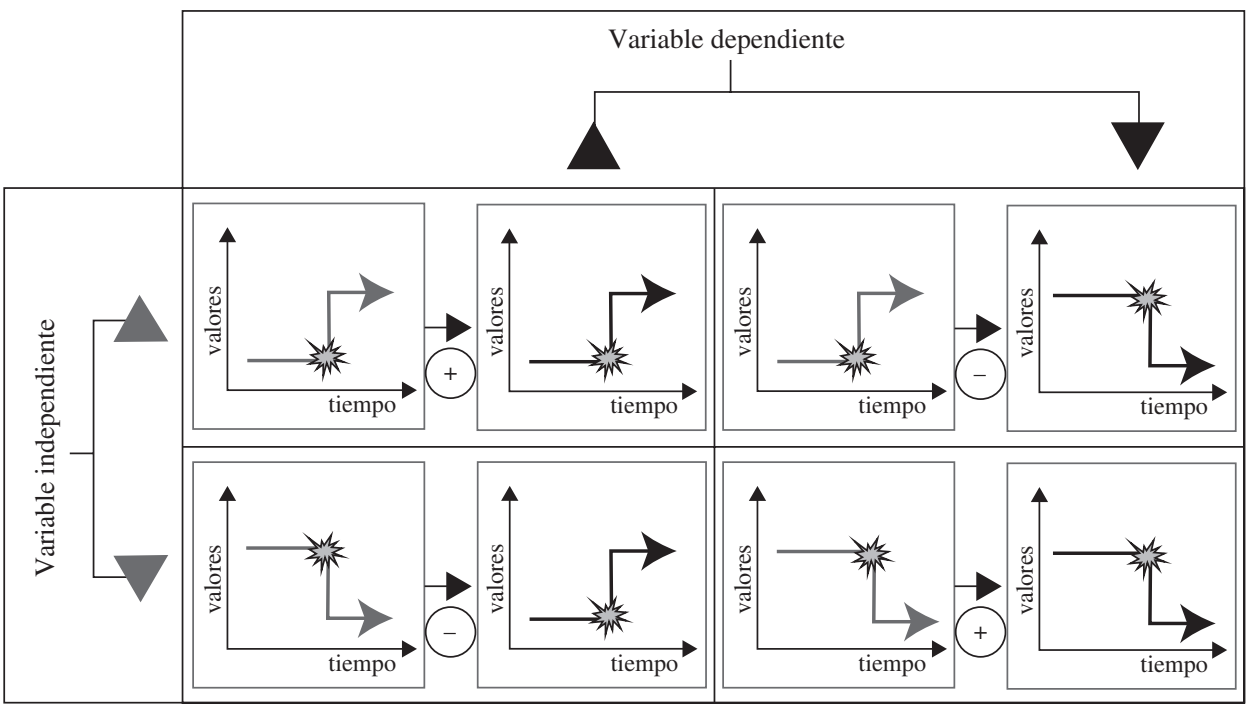

Los diagramas de bucle causal y esta definición y notación de "polaridad" han causado fuertes críticas entre los investigadores (Richardson 1986, 1997; Warren 2004): circulan diferentes definiciones, junto con diferentes notaciones.

La definición "popular" es simple de recordar pero no es enteramente correcta. Un ejemplo recurrente de sus críticos es que, "Natalidad $\rightarrow(+)$ Población" significaría "cuando la natalidad baja, entonces baja la población", lo que parece un contrasentido. Los nacimientos son un flujo entrante o positivo: pueden aumentar la población, pero no disminuirla. Este reclamo es coherente con la definición "popular". Sin embargo, nosotros no ponemos en duda los diagramas causales como lenguaje, sino que esta misma definición, es decir, la manera de usar el lenguaje.

La definición "completa" de polaridad. Para analizar este caso, tenemos que usar otra definición -la "completa"- de polaridad, a saber:

- Polaridad positiva: cuando la variable independiente cambia en un sentido (con signo positivo/negativo), entonces desde este momento en adelante, los valores de la variable dependiente serán más altos/bajos de lo que habrían sido (símbolo “+”);

- Polaridad negativa: cuando la variable independiente cambia en un sentido (con signo positivo/negativo), entonces, desde este momento en adelante, los valores de la variable dependiente serán más bajos/altos de lo que habrían sido (símbolo “-”).

En este sentido, siguiendo el ejemplo anterior: "cuando la natalidad sufre una baja, a partir de este momento la población será más baja de lo que habría sido sin tal 
evento". Al usar la definición completa, desaparece el problema. Esto ya fue admitido por Richardson (1997), pero llama la atención que Warren (2004) en su crítica de los diagramas causales, usa la definición popular.

Hay que considerar que la población está sujeta a la influencia de otras variables, por ejemplo, las muertes. Cuando mueren personas, quedan menos personas vivas. Entonces desde el momento cuando disminuyen los nacimientos (y las muertes no cambian) observaremos efectivamente que la población disminuye (baja): algunos de los que mueren no han sido remplazados por los que nacen.

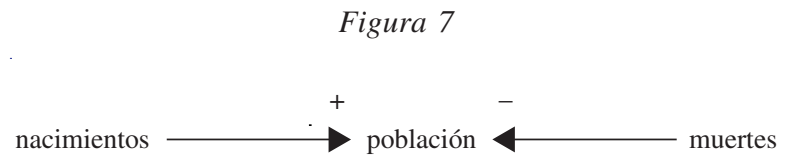

Algo similar pasa en otras circunstancias, por ejemplo cuando en un país la oposición alega que la política de empleo del gobierno ha fallado (porque sigue siendo demasiado alto el desempleo) y el gobierno responde que no es verdad porque gracias a su política el desempleo es menos grave de lo que habría sido sin ella, punto que será discutido en la sección sobre educación (p. 146).

Cuando discutimos las relaciones causales entre variables, tenemos que dirigir nuestra atención a la diferencia entre "lo que fue" y "lo que habría sido". Ello es el resultado de que las dos variables de nuestro modelo participan también en otras relaciones causales: son impactadas por variables que quedaron excluidas del modelo. Esta exclusión nos puede cegar en cuanto a los efectos de estas variables. Si se usa la definición "completa", no hay ningún problema, ya que la conducta general, en cierto modo epifenómeno de las variables excluidas, permite expresar sus efectos. ¿Por qué entonces sigue siendo tan popular la definición incorrecta, tanto en publicaciones de vulgarización (Senge et al. 1995; Senge et al. 2000) como en medios de investigación (Warren 2004)?

Ceteris paribus y variables implícitas. Esto nos conduce a considerar la noción de ceteris paribus; usualmente las definiciones de causalidad/polaridad se acompañan por la cláusula ceteris paribus: "toda otra cosa siendo igual". Se asume además que en el modelo (representado por el diagrama) están consideradas todas las variables (las "todas otras cosas") que influyen en la variable dependiente. Entonces se mantienen iguales todas las variables menos una.

Sin embargo, acabamos de constatar que por reducir la reflexión a solamente dos variables, "todas otras" variables quedaron fuera del modelo. ¿Qué pasa entonces con la condición del ceteris paribus?

Usualmente pensamos que las variables conservan un determinado valor absoluto. En nuestro ejemplo de la población, mantendríamos igual el número de muertes; luego observamos lo que pasa con población cuando cambia el número de nacimientos. Pero: en el ejemplo inicial no se considera el número o tasa de muertes. No podemos mantener igual una variable que no aparece en el modelo/diagrama. Resulta interesante 
constatar que incluso en los escritos que critican los diagramas causales, la discusión se enfoca en solamente dos variables, dejando las otras en lo implícito.

Podemos sospechar que cuando un tomador de decisiones reflexiona para llegar a determinar un curso de acción o una política de decisiones, enfocará su atención a las mismas dos variables. ¿Qué pasa entonces con las otras variables y con el ceteris paribus?

Aparentemente la cláusula se tiende a aplicar a población; se puede inferir esto desde la misma crítica: se dice que la población no puede bajar por causa de los nacimientos, y no se agrega pero la población sí puede bajar debido a la influencia de otras variables (que mantendremos iguales). Todas las otras variables que influyen en la variable dependiente, al mantenerse iguales, causan (colectivamente) una "conducta general" de la variable dependiente. Ceteris paribus debe entonces significar que la "conducta general de la variable dependiente se mantiene igual".

De esta manera, podemos mantener el estilo sistémico de nuestro proceder y a la vez permitir que no se representen todas las variables en el diagrama: en cualquier situación concreta, los tomadores de decisión tendrán acceso a esta "conducta general", que sea desde bases de datos, informes estadísticos o la opinión de expertos.

Por esta razón, creemos que la crítica de Warren (2004) -los diagramas causales son fundamentalmente erróneos (por la razón explicada anteriormente) porque no hacen la diferencia entre variables de flujo y de acumulación- no se puede sostener. Usados con la definición "completa", y cuidando la aplicación de la cláusula ceteris paribus en forma de "conducta general", el usuario del enfoque de diagramas causales no está en peligro de engañarse.

Las variables explícitas no son siempre estacionarias. Si las variables pueden tener una "conducta general", entonces tenemos que reconocer que al reflexionar con un diagrama causal asumimos implícitamente el supuesto de que las variables no cambian salvo cuando interviene el evento que estamos analizando.

Hasta ahora hemos usado solamente ejemplos donde las variables mantienen un mismo valor constante, excepción hecha para el evento de cambio. El que el cambio -causa o efecto- ocurra en precisamente un instante y que en todo el resto del tiempo las dos variables estén estáticas, es un supuesto que, a raíz de los argumentos expuestos, debe ser revisado.

Acabamos de ver que variables como población o desempleo tienen un comportamiento general que resulta de múltiples influencias y sería razonable asumir que no son estáticas. Sin embargo, ¿qué pasa cuando reflexionamos sobre un asunto concreto?

Cuando pensamos en casos como "si el Banco Central baja la tasa de interés, entonces la construcción de viviendas aumenta", la baja interviene en un determinado momento, ¿no damos por sentado que antes, la tasa de interés y la construcción de viviendas han sido constantes? Pensamos "la construcción ES muy baja, cambiemos esto" y la tasa de interés se piensa estática también. Pensamos que las cosas SON, no que DEVIENEN.

Si nos limitamos deliberadamente a comportamientos lineales, entonces cuando analizamos dos variables -y sin importar el evento por el momento- cada una de ellas puede tener una de tres "conductas generales" (o pendientes) lineales: nula, positiva o negativa. 
Para describir esto en nuestro discurso sobre las dos variables introducimos el código "conducta general variable independiente/dependiente", con los siguientes valores posibles:

- "plana" ("s" para "same” en inglés)

- "sube" ("u" para "up" en inglés)

- "baja" ("d" para "down" en inglés)

Cuando interviene un evento, el cambio que gatilla en una variable con pendiente positiva o negativa es un cambio adicional. En estos casos, hablar de baja o de sube es ambiguo, ya que no queda claro si esto se refiere a la conducta general de la variable antes del evento o al cambio gatillado por el evento: hay "cambio" en dos niveles diferentes. Entonces se hace conveniente representar siempre la prolongación de la conducta original (la que habría sido sin el evento) junto con la línea de comportamiento en el tiempo de cada variable. Por lo tanto, para un evento de cambio "sube", tenemos que considerar las siguientes posibilidades:

\section{Figura 8}

"Lo que fue" versus "lo que habría sido"

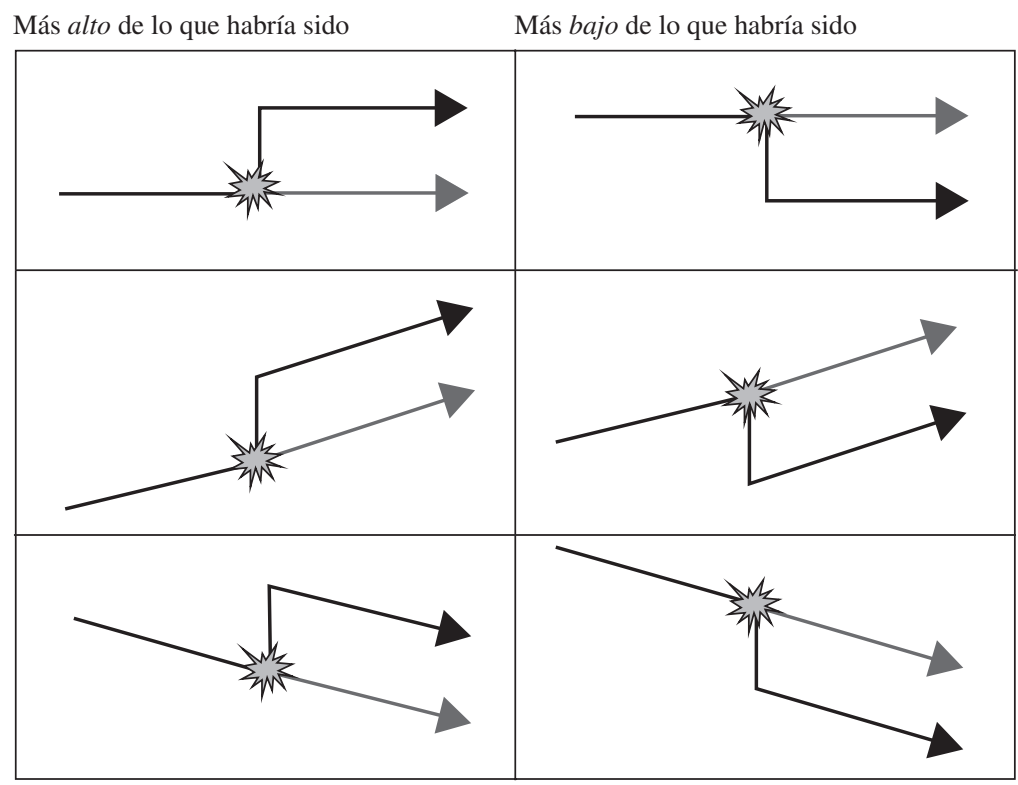

Adoptemos una simplificación adicional: solamente analizaremos casos de cambio que resulten en un desplazamiento vertical, excluyendo por el momento cambios de pendiente. 
En cada uno de estos 3 casos, el desplazamiento equivale a que en cada instante después del evento, el valor de la variable es superior al que habría tenido sin el evento. Análogamente, un evento que baja tiene 3 posibilidades. En cada instante después del evento el valor de la variable es inferior al que habría tenido sin el evento.

Constatemos por ahora que la definición "completa" de polaridad facilita tomar en consideración que en un mundo dinámico las variables pueden estar en cambio antes y después de que un evento intervenga, y entonces los cambios inducidos son cambios adicionales: "más altos/bajos de lo que habría sido" expresa este hecho adecuadamente.

Como resultado de esta manera de ver, las 4 configuraciones presentadas tradicionalmente son solamente casos especiales de un conjunto mucho más extenso de configuraciones. De entrada, la variable independiente y la variable dependiente pueden estar en cada una de las tres posibilidades de pendiente (positiva, nula o negativa), lo que nos genera 9 combinaciones. Para cada una de estas, existen las 4 configuraciones básicas (sube-sube, sube-baja, baja-sube, baja-baja); tenemos pues 36 configuraciones.

\section{Figura 9}

36 configuraciones para dos polaridades

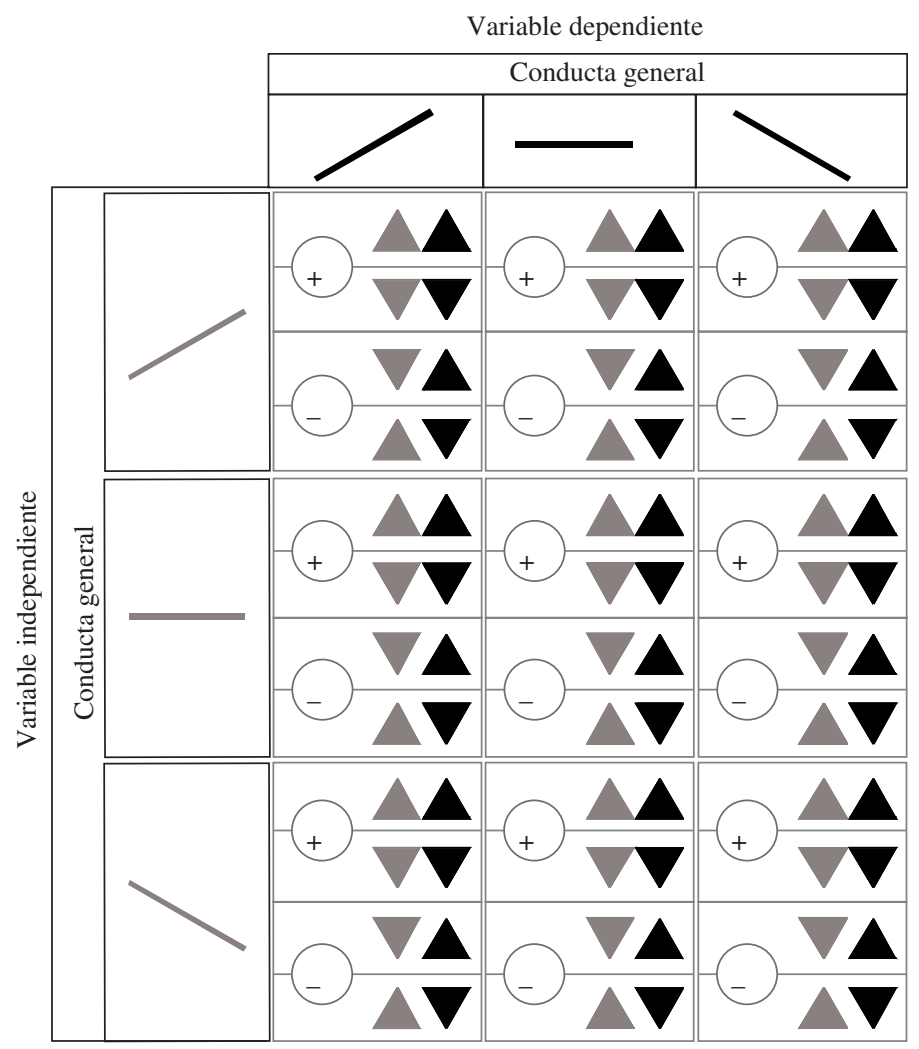


Como la figura lo ilustra, el conjunto de cuatro (4) configuraciones para cada una de las nueve (9) combinaciones es complejo y requiere de concentración para "pensarlo". Recordemos que esto es todavía una simplificación: el número de configuraciones sería aún más grande si admitimos el cambio de pendiente (en este caso, se multiplicaría por 4, llegando a 144 configuraciones) y cuando admitimos conductas no lineales crece aún más. Sin embargo, nuestro punto principal se puede argumentar sin necesitar esta ampliación: "Conjeturamos que las personas tienden a pensar en las variables en términos estáticos (no dinámicos) y que por lo tanto su mente se focaliza en las 4 configuraciones "simples" donde tanto la variable independiente como la dependiente son planas (la zona del centro en la figura previa)".

Si esto es así, entonces la preferencia para la definición "popular" es coherente: esta definición permite clasificar correctamente cada una de las 4 configuraciones "simples". Ello explicaría también por qué la mayoría de personas encuentra la definición "completa" compleja: se refiere a un mundo dinámico, en el cual existen -además de las 4 "simples"- 32 (o más) configuraciones "complejas". Encontramos entonces en el caso de la polaridad la complejidad dinámica, consecuencia de la limitación de la mente humana (y a la cual la dinámica de sistemas pretende aportar).

Podemos organizar las configuraciones en 4 grupos de cuadrantes, que corresponden a diferentes arreglos de conducta general:

\section{Figura 10}

9 cuadrantes para las configuraciones

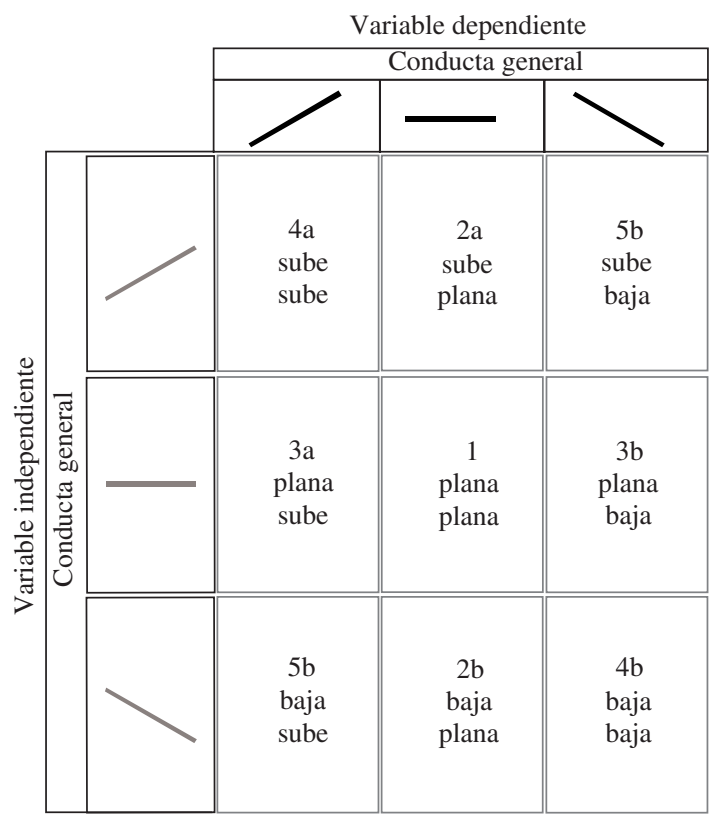


La definición popular permite clasificar adecuadamente solamente a las 4 configuraciones del cuadrante 1; en cambio, la definición "completa" cubre también las configuraciones de los 8 cuadrantes restantes.

\section{Figura 11}

La definición "popular" para el cuadrante 1, la definición "completa" incluye también los otros cuadrantes
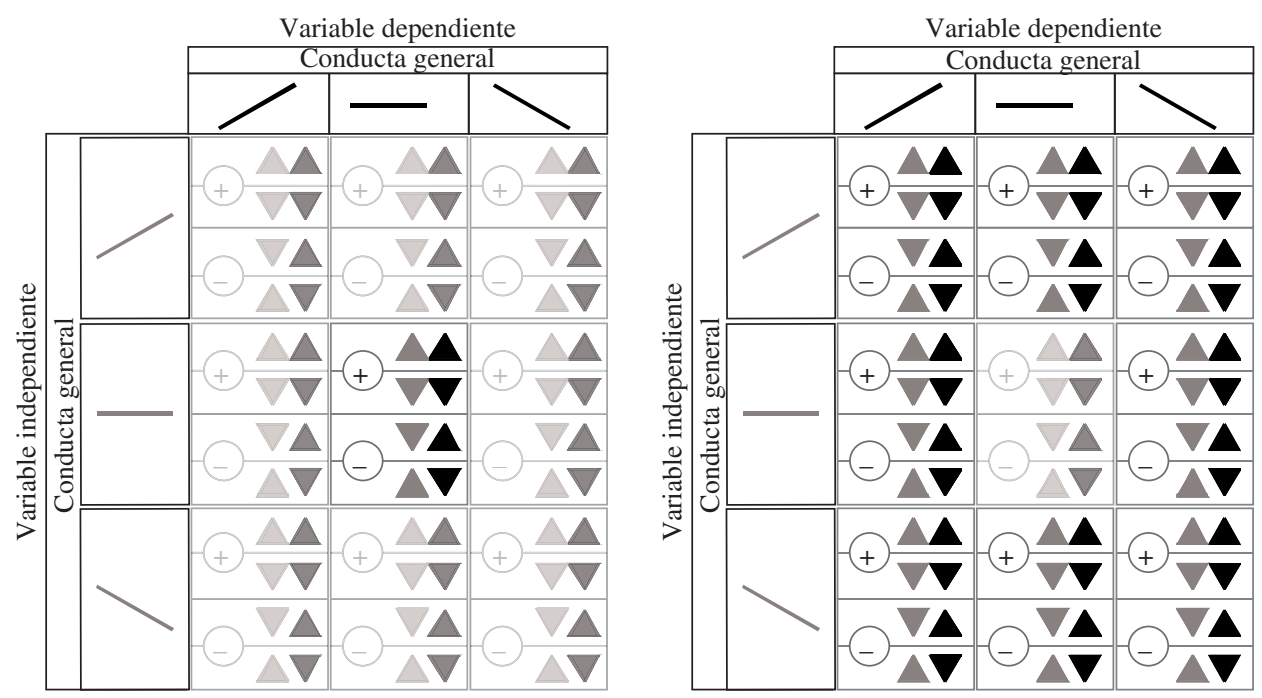

Sostenemos que el modelo conceptual de las configuraciones es a la vez un espacio de investigación y un fundamento para el desarrollo de artefactos educacionales. Ambas temáticas serán elaboradas a continuación.

\section{INVESTIGACION DEL PENSAMIENTO CAUSAL: ESTATICO VERSUS DINAMICO}

El pensamiento estático ha sido tratado en dos formas dentro del contexto de la dinámica de sistemas. Sterman (1989) investigó la sorprendente falla de ejecutivos y estudiantes del MIT al manejar una tienda de distribución de bebidas en el contexto de un juego de mesa, donde cada participante debe vender bebidas a su comprador y hacer pedidos a su proveedor para no quedar sin aprovisionamiento. Resulta que cuando 4 de estas tiendas -botillería, mayorista, distribuidor y fábrica- operan en cadena, generan oscilaciones endógenas que se van amplificando (fenómeno hoy en día denominado "efecto látigo"). Sterman mostró que en el pensamiento de los sujetos ocurrió un efecto de "anchoring and adjustment" usando la cantidad inicial en bodega como un "ancla" estática. 
Posteriormente apareció que en situaciones que involucran la interacción de variables de acumulación y variables de flujo, las personas comunes se equivocan sistemáticamente en apreciar en forma correcta la relación entre ambas (Booth-Sweeny y Sterman 2000; Ossimitz 2002; Schaffernicht 2006).

Moxnes $(1998,2000,2004)$ encontró que algunos fenómenos de sobreexplotación de recursos naturales no deben ser atribuidos al problema de los bienes públicos o "commons", donde la falta de señal de escasez conduce a la sobreexplotación. Más bien, se sospecha un problema en el pensamiento de los tomadores de decisión. Por ejemplo, cuando se trata de administrar una manada de renos que se alimentan de musgo en un territorio limitado hay una capacidad máxima del territorio para soportar los renos: si hay más renos, comen demasiado y se quitan la base de su subsistencia. Si hay menos renos, las ganancias serán reducidas. El administrador controla el tamaño de la manada de renos. Los experimentos dan señales de que en la mente de los sujetos la transmisión del efecto de un cambio de la cantidad de renos a la cantidad de musgo, el tiempo no juega ningún rol, es como si el ajuste se diera instantáneamente.

Figura 12

¿Menos renos -> más musgo?

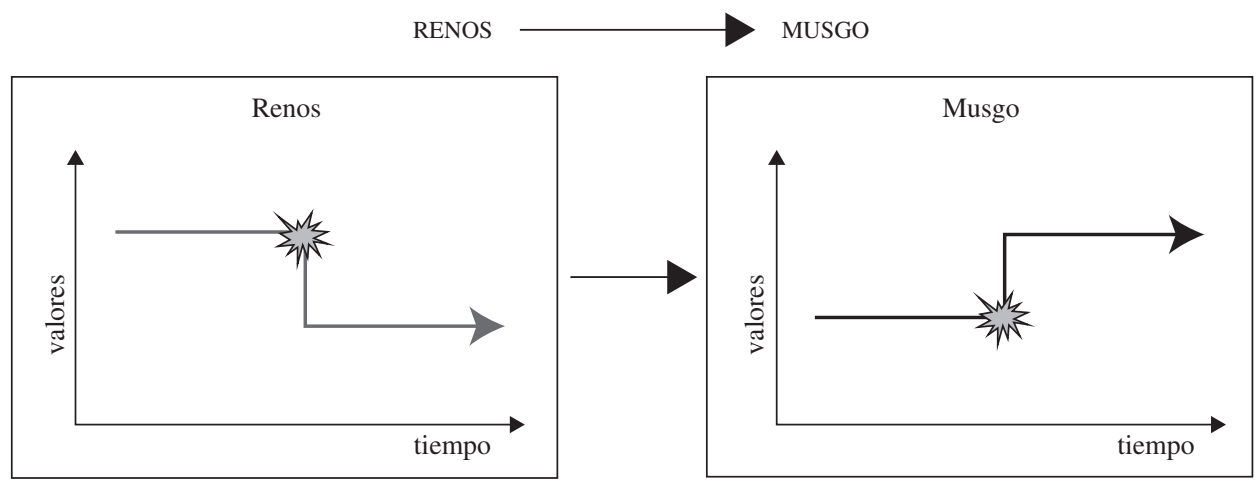

Resulta que este modelo es demasiado reducido. Hay variables importantes que quedaron excluidas: la cantidad de musgo que comen los renos (que depende de la cantidad por reno y del número de renos) y la cantidad de musgo que se regenera (depende de la tasa de regeneración y la cantidad de musgo). Entonces el efecto que obtiene una reducción de la cantidad de renos puede ser todo desde una disminución de la reducción hasta una vuelta al crecimiento, dependiendo de cuántos renos se sacan de la manada. En este sentido, Moxnes habla de pensamiento estático.

En nuestro caso, también se puede decir que en el pensamiento detrás de la definición "popular", el tiempo no juega un rol, pero es en un sentido diferente. No hablamos de efecto instantáneo, sino que de la detención de la conducta general que inducen las "otras" variables (excluidas del modelo) en la variable dependiente. 
Se abre entonces una tercera dimensión a la noción de pensamiento estático. Esta dimensión deberá ser investigada de manera empírica, por lo cual proponemos un conjunto de preguntas de investigación junto con una sistematización de las configuraciones que permite realizar un trabajo experimental.

\section{PREGUNTA 1}

La conjetura arriba presentada significa que la conducta general de las variables incide en el esfuerzo cognitivo que las personas deben hacer para decir si en una determinada situación se trata de polaridad positiva o negativa. En otras palabras, cuando la conducta general de las variables no es estática (cuadrante 1), ¿es más fácil que cuando tiene pendiente no nula (los demás cuadrantes)? Un primer experimento ayudará a corroborar esta hipótesis:

H1: la definición "popular" conduce a un rendimiento correcto en el cuadrante 1 y pobre en los demás cuadrantes, mientras que la definición "completa" conduce a un rendimiento bueno y constante sobre todos los cuadrantes.

Para esto, un grupo de sujetos deberá clasificar (indicar la polaridad) en diversas situaciones del cuadrante 1 y de los demás cuadrantes con la ayuda de la definición "popular", mientras un grupo control abordará las mismas situaciones, pero con la definición "completa". En estas pruebas, se toma como rendimiento la combinación entre la corrección de la clasificación y el tiempo usado para clasificar. Si el rendimiento del primer grupo es mejor en las situaciones del cuadrante 1 (en comparación con los demás cuadrantes), pero en el grupo de control el rendimiento de los otros cuadrantes es tan bueno como en el cuadrante 1, entonces la hipótesis se aceptará.

\section{PREGUNTA 2}

Luego tenemos que reconocer que a veces una de las dos variables es estática y la otra tiene una pendiente no nula. Surge entonces la pregunta ¿es más difícil pensar sobre la variable independiente o la dependiente? Por ello se formula la segunda hipótesis:

\section{H2: es más difícil pensar con la variable dependiente que con la independiente.}

Corroborar esta hipótesis es posible con un experimento usando las mismas situaciones; esta vez, no es necesario distinguir entre los dos grupos que usan definiciones distintas, ya que la pregunta no se refiere a la definición de polaridad. Se compara el rendimiento en las situaciones del cuadrante 2 (variable independiente con pendiente no nula, variable dependiente estática) con el rendimiento para el cuadrante 3 (al revés). Si el rendimiento del cuadrante 2 supera el del cuadrante 3, se dará por corroborada la segunda hipótesis.

\section{PREGUNTA 3}

El lector habrá observado que en la mitad de los casos de pendiente no nula, la pendiente es positiva y en la otra mitad es negativa. ¿Pensar con una variable en 
crecimiento es diferente que pensar con una que baja? Formulamos así la tercera hipótesis:

H3: es más difícil pensar con una variable con pendiente negativa que con una variable con pendiente positiva.

De acuerdo a esto, el rendimiento de los cuadrantes con una "a" (donde la pendiente es positiva: $2 \mathrm{a}, 3 \mathrm{a}, 4 \mathrm{a}$ y $5 \mathrm{a}$ ) debería ser superior al rendimiento en los cuadrantes con una "b" (2b, 3b, 4b y 5b).

\section{PREGUNTA 4}

La polaridad es la relación entre los signos de cambio en las dos variables. Hemos visto que para cada tipo de polaridad hay dos posibilidades (Figura 6, p. 135). En el caso de la polaridad positiva, tenemos que tratar o con dos variables que aumentan o con dos variables que bajan. ¿Puede ser que sea más difícil pensar sobre el segundo caso?

H4: es más difícil pensar sobre polaridad positiva con dos variables que bajen que con dos variables que aumenten.

Para responder a esta interrogante, se comparan los rendimientos en situaciones de polaridad positiva donde ambas variables aumentan con los rendimientos en situaciones de la misma polaridad donde ambas variables bajan.

\section{PREGUNTA 5}

Si fue razonable preguntar sobre la relativa dificultad de pensar con variables que cambian con signo positivo o negativo, también es posible que haya una diferencia entre el rendimiento observable entre situaciones de polaridad positiva y negativa:

H5: es más difícil pensar con polaridad negativa que con polaridad positiva.

Para llegar a aclarar este aspecto se comparan los rendimientos de las situaciones con polaridad positiva a los de las situaciones con polaridad negativa, tomando en consideración situaciones de todos los cuadrantes. En el caso que la hipótesis 4 haya sido corroborada, se considerarán solamente las configuraciones "sube, sube" para la polaridad positiva.

Sistemática para un experimento. Hemos elaborado una tabla de clasificación de las 36 configuraciones, en la cual se distinguen las diferentes conductas generales (figura 10, p. 140), la polaridad del vínculo causal y los signos de cambio de ambas variables (tabla 1, p. 133).

El experimento contempla un conjunto de tareas de clasificación que cubre las 36 configuraciones, donde en cada tarea se invita al sujeto a decidir si la situación presentada corresponde a un vínculo causal de polaridad "positiva" o "negativa" (dejando la posibilidad de decidir "no se puede saber" y“"no lo sé”). 
Tabla 2

Sistemática de configuraciones

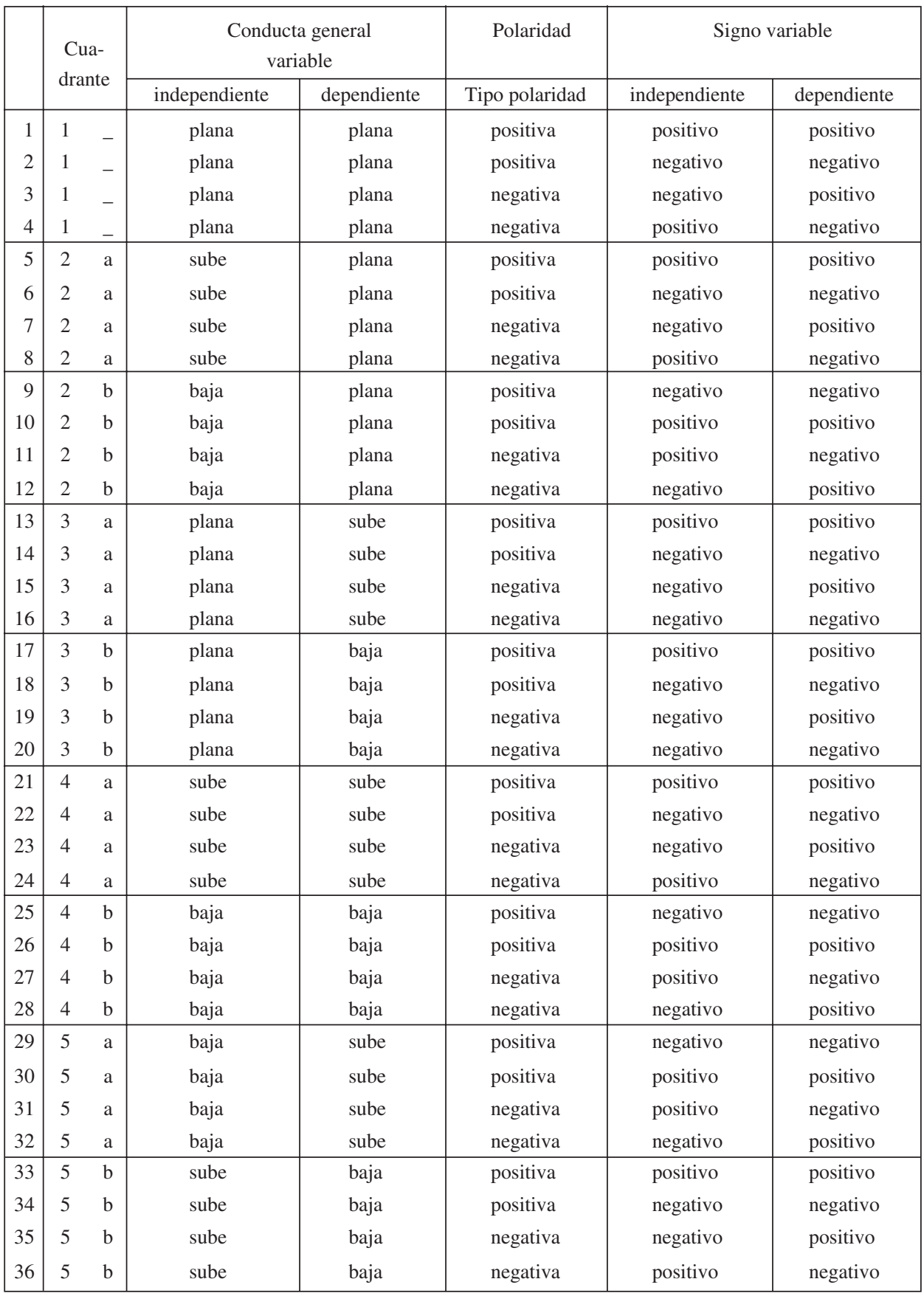


Figura 13

Una tarea de clasificación ejemplar

Clasifique la siguiente situación

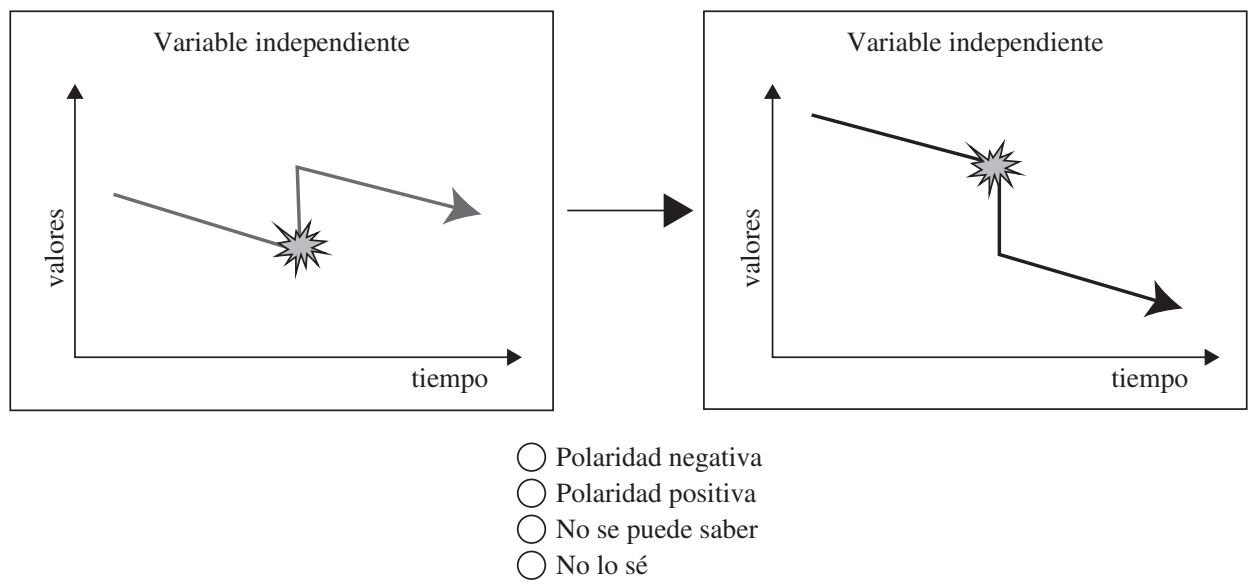

Se forman dos grupos de sujetos: uno para trabajar con la definición "popular", el otro para trabajar con la definición "completa". Asegurando un número de ejemplares igual para cada configuración, el conjunto de respuestas permitirá responder a las preguntas de investigación planteadas.

\section{EDUCACION PARA EL PENSAMIENTO CAUSAL DINAMICO}

El pensamiento causal es un tema relevante para la mayoría de ámbitos de educación, ya sea al nivel escolar o universitario. Por donde aparecen argumentos y por donde hay que resolver problemas, la reflexión consciente usa atribuciones causales.

Algunos ejemplos prominentes aparecen en la vida pública. En muchos países, el Estado está realizando reformas en los sistemas de educación o de salud; en general la oposición de turno acusa al gobierno de turno de que los procesos de reforma fracasan o no avanzan lo suficientemente rápido. Los temas de la criminalidad y del desempleo son escenario de debates similares. Para razonar bien sobre estos asuntos, la pregunta acerca del efecto logrado por la acción del Estado debe tomar en cuenta la conducta general de las variables que se discuten, ya que el Estado no es la única influencia en este caso. Esto es lo que en general no se hace. Por ejemplo, no se debe preguntar si el Estado logró reducir el desempleo; la pregunta correcta es si el desempleo habría sido peor sin la acción del Estado.

¿Cuántas de estas acusaciones se basan en un pensamiento estático, y cuántos ciudadanos dejan influir su juicio por esta vía?

Un adecuado pensamiento causal -dinámico- aparece entonces como recurso para el pensamiento crítico. Si no se forma intuitivamente, ¿cómo se puede enseñar? 
Nuestra propuesta se refiere solamente al aspecto del pensamiento dinámico que hemos discutido aquí; un segundo componente se propondrá a continuación.

Las tareas usadas para la investigación empírica pueden ser reutilizadas con fines educativos, pensando en el uso de computadores para implementar los ejercicios.

\section{Figura 14}

Proceso educativo

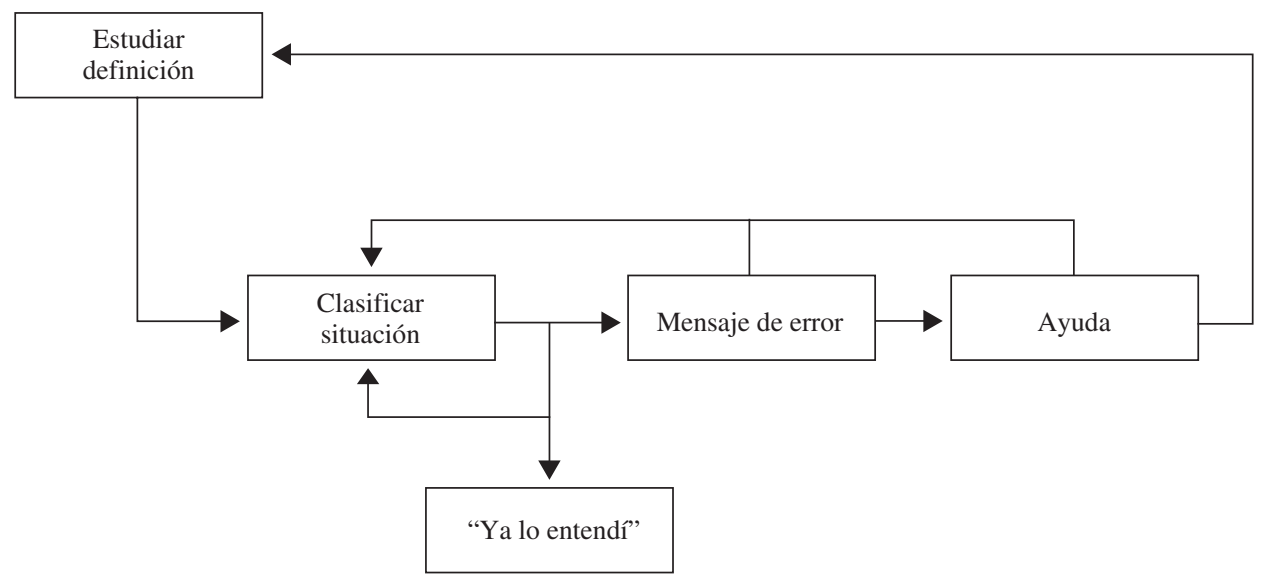

Después de haber estudiado la definición y las explicaciones que la fundamentan, el estudiante empieza a clasificar situaciones. Si comete un error en una de estas tareas, primero se le devuelve un mensaje de error avisando de lo extraño de su elección. Desde este punto, puede regresar a la tarea e intentar clasificarla correctamente, o bien desea obtener ayuda (en forma de ejemplos correctamente clasificados y con justificación). Nuevamente, puede elegir volver a la tarea o expresar su deseo de volver a estudiar la definición.

Al haber clasificado correctamente una situación se le conduce a la siguiente (en orden de complejidad creciente; flechas grises en la figura). La reiterada solución exitosa de tareas conducirá al reconocimiento de que "ya lo entendí", que marca el punto final de este proceso.

A continuación, será recomendable someterse a tareas de resolución de problemas dinámicos, tal como es usado en los experimentos de Moxnes (2004), donde el pensamiento correcto debe pasar la prueba de la acción.

A partir de este nivel, cabe reconocer la existencia de los otros aspectos del pensamiento estático (anteriormente señalado) y recomendar una empresa más general de educación al pensamiento dinámico. Sin reclamar la exclusividad del área para la disciplina de la dinámica de sistemas, es bueno recordar que ya durante los años 90 hubo intentos de conceptualizar el "buen" pensamiento dinámico (Richmond 1993). Sin embargo, este tópico nos conducirá más allá del terreno del presente artículo. 


\section{CONCLUSIONES}

Al presente, se ha concluido una etapa. Hemos elaborado una visualización del tema de los vínculos causales con polaridad que pone énfasis en la existencia de dos niveles de conducta de las variables: uno general y el otro particular a un evento. De esta forma, hemos podido mostrar las implicancias de las dos definiciones diferentes de polaridad:

- la definición "popular" es correcta para las 4 configuraciones donde la conducta general es estática;

- la definición "completa" también es correcta para las 32 configuraciones donde la conducta general no es estática, es decir, donde una o ambas variables tienen una pendiente general no nula.

Explicamos la persistencia de la definición "popular" con que, intuitivamente, las personas prefieren pensar estáticamente sobre las variables. A su vez, ello nos conduce a agregar una nueva dimensión del pensamiento estático a las dos dimensiones ya reconocidas en la literatura.

Hemos formulado un conjunto de 5 preguntas de investigación empírica y una forma de organizarla alrededor de una sistemática de las configuraciones. Creemos que se pueden formular más preguntas y también que el estudio de datos provenientes de diferentes localidades permitirá progresar más rápidamente en este tema. Llamamos a la comunidad a participar de estas investigaciones.

También hemos indicado una forma de usar este trabajo en una orientación de educación. Igual que en la parte investigativa, creemos que la realización de experiencias y la discusión de sus resultados permitirán una acumulación de conocimientos que no puede sino ayudar a progresar en este tema.

Cerramos subrayando la importancia de realizar investigaciones en el ámbito del pensamiento causal dinámico, en el deseo de aportar a la educación de ciudadanos críticos en un mundo de creciente complejidad.

\section{BIBLIOGRAFIA}

Booth-Sweeney, L. y J.D. Sterman (2000). Bathtub dynamics: initial results of a systems thinking inventory. System Dynamics Review 16 (4): 249-286.

Moxnes, E. (1998). Overexploitation of renewable resources: The role of misperceptions. Journal of Economic Behavior \& Organization 37: 107-127.

Moxnes, E. (2000). Not only the tragedy of the commons: misperceptions of feedback and policies for sustainable development. System Dynamics Review 16 (4): 325-348.

Moxnes, E. (2004). Misperceptions of basic dynamics: the case of renewable resource management. System Dynamics Review 20 (2): 139-162.

Pedercini, M. (2006). What's behind the blue arrow? The notion of causality in System Dynamics. Actas del $24^{\text {to }}$ Congreso Internacional de la Sociedad de Dinámica de Sistemas, Nijmegen (Holanda), julio 2006.

Ossimitz, G. (2002). Stock-Flow-Thinking and Reading stock-flow-related Graphs: An Empirical Investigation in Dynamic Thinking Abilities, 2002 System Dynamics Conference. Palermo, Italy (CD). 
Richardson, G. P. (1991). Feedback thought in social sciences and systems theory. Pegasus Communications, 1991.

Richardson, G. P. (1997). Problems in causal loop diagrams revisited. System Dynamics Review 13 (3), (Fall 1997): 247-252.

Richmond, B. (1993). Systems thinking: critical thinking skills for the 1990s and beyond. System Dynamics Review 9 (2), (Summer 1993): 113-133.

Warren, K. (2004). Why has feedback systems thinking struggled to influence strategy and policy? Systems Research and Behavioral Science (Jul/Aug 2004) 21 (4): 331.

Schaffernicht, M. (2006). LaTina - An online system for teaching and learning stock-and-flow thinking skills. Actas del $24^{\text {to }}$ Congreso Internacional de la Sociedad de Dinámica de Sistemas. Nijmegen (Holanda), julio 2006.

Senge, P., R. Ross, B. Smith, Ch. Roberts y A. Kleiner (1995). La Quinta disciplina en la práctica. Granica.

Senge, P., Ch. Roberts, R. Ross, G. Roth y B. Smith (2000). La Danza del Cambio. Norma.

Sterman, J.D. (1989). Modelling managerial behavior: Misperceptions of feedback in a dynamic decision making experiment. Management Science 35 (3): 321-339.

Sterman, J. D. (2000). Busyness Dynamics - systems thinking and modeling for a complex world. John Wiley. 
Las colaboraciones, pedidos, suscripciones y correspondencia deben ser dirigidas a la Dirección de la revista: Oficina de Publicaciones, Facultad de Filosofía y Humanidades, Universidad Austral de Chile, Casilla 567, Fono/Fax: 5663 221275, Valdivia, Chile. E-mail: eped@uach.cl

Las suscripciones incluyen los gastos de envío:

Chile: \$ 2.000. Otros países: US\$ 10.

El canje debe ser enviado a Biblioteca Central, Universidad Austral de Chile,

Correo 2, Valdivia 
ANDROS IMPRESORES

WWW.androsimpresores.cl 
